\title{
24-Month Efficacy of Viscodilation of Schlemm's Canal and the Distal Outflow System with iTrack Ab-Interno Canaloplasty for the Treatment of Primary Open-Angle Glaucoma
}

This article was published in the following Dove Press journal:

Clinical Ophthalmology

\author{
Mark J Gallardo (D) \\ EI Paso Eye Surgeons, PA, El Paso, \\ TX, USA
}

Correspondence: Mark J Gallardo El Paso Eye Surgeons, PA, I20I N Mesa St, Ste G, El Paso, TX, 79902, USA

Tel +l 9155420279

Email gallardomark@hotmail.com
Purpose: This study aimed to evaluate the 24-month efficacy of viscodilation of Schlemm's canal and the distal outflow system with iTrack ab-interno canaloplasty in reducing intraocular pressure (IOP) and antiglaucoma medication use in patients with primary open-angle glaucoma (POAG).

Patients and Methods: This retrospective, comparative, and consecutive case series assessed the 24-month outcomes of iTrack as a standalone procedure (iTrack-alone) or in conjunction with cataract surgery (iTrack+phaco), in cases of mild, moderate or severe POAG. Data were collected at baseline and the 12- and 24-month follow-up visits.

Results: The study included 53 patients (60 eyes) with a mean age of $73.6 \pm 9$ years. For all eyes, a significant reduction in mean IOP was seen at 12 and 24 months, reducing from 20 $\pm 4.9 \mathrm{mmHg}$ at baseline to $13.6 \pm 1.9 \mathrm{mmHg}$ and $13.5 \pm 2.6 \mathrm{mmHg}$, respectively $(P<0.001)$. The reduction in medications was also significant, reducing to $1.12 \pm 1.09$ and $1.7 \pm 1.29$ medications at 12 months and 24 months, respectively, from a baseline of $2.77 \pm 0.91(P<0.001)$. Similar results were achieved whether iTrack was performed as a standalone procedure, or in conjunction with cataract surgery. While the iTrack-alone group started with a higher IOP at baseline, both groups experienced similar and stable IOP reduction of more than $30 \%$ from baseline. The reduction in medication use was also significant in both groups, decreasing from $3 \pm 0.7$ for iTrack-alone and $2.5 \pm 1$.1 for iTrack+phaco at baseline to $2.1 \pm 1.3$ and $1.3 \pm 1.2$ at 24 months, respectively $(P<0.001)$. The 24 -month efficacy outcomes were similar in cases of mild-moderate versus severe glaucoma. No serious adverse events were recorded.

Conclusion: Twenty-four-month follow-up data demonstrate that iTrack ab-interno canaloplasty can effectively reduce IOP and medication dependence, with a good safety profile. These results also demonstrate that iTrack can be effectively performed as a standalone procedure or in combination with cataract surgery, and in all grades of glaucoma severity.

Keywords: MIGS, canaloplasty, viscodilation, intraocular pressure, glaucoma medication, iTrack

\section{Introduction}

Glaucoma is the second leading cause of blindness worldwide, estimated to affect close to 60 million people in $2020 .^{1}$ The main treatment goal for primary openangle glaucoma (POAG) is the reduction of intraocular pressure (IOP) to prevent progressive optic nerve damage and visual field deterioration. Traditionally, the glaucoma treatment algorithm has consisted of topical antiglaucoma medications, 
followed by laser treatment and penetrating filtration surgeries such as tube shunts and trabeculectomy. ${ }^{2}$

Despite their high degree of efficacy, antiglaucoma medications are not without negative side effects such as toxicity to the eye and increased risk of ocular surface disease. ${ }^{3}$ They also suffer from a poor rate of compliance and impact negatively on patient quality of life. , $^{3,4}$

In the last 10 years, the development of minimally invasive glaucoma surgeries (MIGS) has transformed the landscape in glaucoma management, allowing surgeons to perform less invasive surgical procedures that may reduce or eliminate the need for antiglaucoma medications, as well as delay the need for more invasive surgical procedures such as tube shunts and trabeculectomy.

The various MIGS procedures attempt to improve outflow resistance by either increasing trabecular outflow, increasing uveoscleral outflow, increasing subconjunctival outflow, or decreasing aqueous production. The majority of MIGS, including stent-based procedures that rely on the insertion of an implant through the trabecular meshwork or in Schlemm's canal, are focal in their approach. It is understood that the glaucomatous eye is associated with a pathology of the entire outflow system, however, including the distal portion. ${ }^{5}$ Indeed, looking beyond the proximal outflow system, additional points of resistance may reside in Schlemm's canal, collector channels, and the scleral plexus. In the glaucomatous eye, greater contractility of Schlemm's canal results in increased stiffness, which increases outflow resistance. ${ }^{6-8}$ Further, the canal of a glaucoma patient tends to be shorter, more narrowed, and often collapsed. ${ }^{6-8}$ Another significant point of resistance is the frequent herniations of the trabecular meshwork/ Schlemm's canal endothelium obstructing up to $90 \%$ of collector channels in POAG eyes, as opposed to only $15 \%$ in non-glaucomatous eyes. ${ }^{9}$

iTrack ab-interno canaloplasty (Nova Eye Medical Inc., Fremont, California) (previously reported as $\mathrm{ABiC})^{10}$ is a stent-free MIGS that combines 360-degree catheterization of Schlemm's canal and viscodilation to comprehensively remove the points of glaucoma blockage in all parts of the conventional outflow pathway, both proximal and distal. Specifically, the procedure targets the three main sites of outflow resistance in the conventional outflow pathway: the trabecular meshwork, Schlemm's canal, and the distal collector channels. ${ }^{11-14}$

iTrack acts mechanically, by breaking adhesions in Schlemm's canal and separating herniations into collector channels via the 360-degree catheterization of the canal, and biomechanically, through a process of pressurized viscodilation that enlarges Schlemm's canal and dilates the distal outflow system. ${ }^{11-14}$ During viscodilation, viscostatic pressure caused by delivery of hyaluronic acid (HA)based ophthalmic viscosurgical device (OVD) stretches the trabecular meshwork, with possible creation of microperforations into the anterior chamber. ${ }^{13,14}$ The delivery of OVD, therefore, dilates Schlemm's canal and improves the flow of aqueous to the collector channels, ${ }^{14}$ further complementing the mechanical opening of Schlemm's canal achieved via the circumnavigation of the iTrack microcatheter through the canal.

Featuring a $250-\mu \mathrm{m}$ atraumatic bulbous tip, the iTrack canaloplasty microcatheter (Nova Eye Medical Inc., Fremont, California) is designed to bypass collector channel ostia and push trabecular meshwork herniations out of the ostia with minimal tissue trauma. An illuminated fiber optic tip provides continuous location feedback, preventing misdirection into the collector channel ostia or the suprachoroidal space.

Performed via an ab-interno approach to Schlemm's canal, iTrack spares conjunctival manipulation. It is also an angle-sparing and tissue-sparing procedure, allowing other additional glaucoma procedures to be performed in the future, if necessary.

Here, we report 24-month efficacy outcomes of iTrack ab-interno canaloplasty both as a standalone procedure (iTrack-alone) and in combination with cataract surgery (iTrack + phaco) in cases of mild, moderate and severe glaucoma.

\section{Patients and Methods \\ Study Design}

In this retrospective analysis of a consecutive case series, we evaluated the efficacy of iTrack ab-interno canaloplasty in reducing IOP and medication dependence, both as a standalone procedure (iTrack-alone) and in combination with cataract surgery (iTrack + phaco) in POAG patients.

Baseline ophthalmic examination included history of glaucoma, medication use, IOP, best-corrected distance visual acuity (CDVA), gonioscopy, slit lamp, and fundus examination.

Primary endpoints included mean IOP and mean number of antiglaucoma medications at 12 and 24 months (percent and number of medications). Secondary endpoints included mean reduction in IOP and medication 
dependence based on glaucoma severity, VA outcomes and surgical and postsurgical complications.

All surgeries were performed at an outpatient surgical facility in El Paso, Texas, by a single surgeon (MJG) between September 2014 and June 2016. This study was performed in compliance with the ethical principles of the Declaration of Helsinki, Good Clinical Practice (GCP), and ISO 14155:2011 as well as applicable local regulations. The study was granted a waiver of consent as it was retrospective in nature and it did not include any patient identifiers. A procedure consent form was completed for all patients.

\section{Patient Selection}

Inclusion criteria were adult POAG patients (18 years of age or older) with a diagnosis of mild-moderate $(n=29)$, or severe glaucoma $(\mathrm{n}=21)$, as per the Hodapp-ParishAnderson method of classification. ${ }^{16}$ Disease severity was determined based on mean deviation (MD) from Humphrey visual field (HVF) testing: $\mathrm{MD} \geq 0 \mathrm{~dB}$ (mild), -0.01 to $-12 \mathrm{~dB}$ (moderate), and $\leq-12 \mathrm{~dB}$ (severe), and confirmed evidence of characteristic changes to the optic nerve consistent with glaucomatous optic neuropathy and thinning of the retinal nerve fiber layer on ocular coherence tomography (OCT). Patients were selected based on whether the angle was healthy, as per assessment with gonioscopy, with treatment limited to those with a homogenously pigmented trabecular meshwork without variegation or excessive pigmentation. Patients with any sign of angle disease, such as peripheral anterior synechiae, goniosynechiae or angle recession, were excluded from the study. Additional exclusion criteria included patients who had undergone laser trabeculoplasty (LTP) within 6 months of surgery or other angle-based microinvasive procedures. Patients with neovascular disease, uveitis, peripheral anterior synechiae, and developmental or other forms of secondary glaucoma, such as steroidinduced glaucoma, were also excluded.

\section{Surgical Technique}

iTrack ab interno canaloplasty was performed as previously described. ${ }^{10}$ During the procedure, the microcatheter is inserted through a small goniotomy into Schlemm's canal. Next, the microcatheter circumnavigates the entire 360 degrees of Schlemm's canal. If an obstruction in the canal prevents the passage of the microcatheter, the microcatheter is withdrawn from the eye and a second paracentesis is created in order to perform the procedure from the opposite direction. Once the iTrack microcatheter has completed circumnavigation it is slowly withdrawn. During the withdrawal of the microcatheter, precisely regulated aliquots of high-molecularweight hyaluronic acid (HA)-based OVD (ophthalmic viscosurgical device) are delivered into Schlemm's canal via a manually operated viscoinjector device, with approximately 9 notches delivered per quadrant over the full 360 degrees of Schlemm's canal. Once this process of viscodilation is complete, the iTrack microcatheter is withdrawn from the eye. In the iTrack+phaco group, cataract surgery was performed prior to ab-interno canaloplasty.

The post-operative regimen varied based on the patient's prescription drug plan but typically involved a topical steroid like prednisolone acetate $1 \%$, a nonsteroidal anti-inflammatory and a fourth generation fluoroquinolone. The antibiotic and NSAID were removed after the first week and the prednisolone was reduced to twice a day dosage for 2 weeks then stopped. Glaucoma medications were stopped on the postoperative day 1 visit and reinitiated if pressures elevated above target.

\section{Statistical Analysis}

All continuous data are represented as mean \pm SD. A repeated-measures ANOVA test was used to compare mean IOP and number of medications between groups over time followed by a post hoc Tukey's test for multiple comparisons across the visits. $P$ values $<0.05$ were considered significant. A $t$-test was performed to compare the $\%$ reduction in IOP and number of medications between groups.

\section{Results}

\section{Patients' Demographics and Preoperative Characteristics}

The characteristics and demographics of the patients are summarized in Table 1. The study cohort included 60 eyes of 53 patients presenting with POAG who met inclusion and exclusion criteria and completed the preoperative visit. The study cohort consisted of patients from Hispanic (73.5\%), black (5.5\%), or white $(21 \%)$ ethnicity, across the glaucoma stages of mild (38\%), moderate $(17 \%)$ and severe $(38 \%)$. The average age was $73.6 \pm 9$ years (range 52-91). Patients had an overall mean preoperative IOP of $20.7 \pm 4.9 \mathrm{mmHg}$ across all eyes on $2.77 \pm 0.9$ medications $(\mathrm{n}=60)$ (Figures 1 and 2). 
Table I Preoperative Patient Demographics and Characteristics for All Eyes

\begin{tabular}{|c|c|c|c|}
\hline & \multicolumn{3}{|c|}{ Preoperative Demographics } \\
\hline & & $\mathbf{n}$ & Percentage \\
\hline \multirow[t]{3}{*}{ Gender } & Female & 32 & 60 \\
\hline & Male & 21 & 40 \\
\hline & $\begin{array}{c}\text { Total no. of } \\
\text { patients }\end{array}$ & 53 & 100 \\
\hline \multirow[t]{4}{*}{ Ethnicity } & Black & 3 & 5.5 \\
\hline & Hispanic & 39 & 73.5 \\
\hline & White & 11 & 21 \\
\hline & $\begin{array}{l}\text { Total no. of } \\
\text { patients }\end{array}$ & 53 & 100 \\
\hline \multirow[t]{3}{*}{ Eye } & Left & 28 & 47 \\
\hline & Right & 32 & 53 \\
\hline & Total no. of eyes & 60 & 100 \\
\hline Glaucoma & Mild & 23 & 38 \\
\hline \multirow[t]{5}{*}{ Stage } & Moderate & 10 & 17 \\
\hline & Severe & 23 & 38 \\
\hline & Missing & 4 & 7 \\
\hline & Total no. of eyes & 60 & 100 \\
\hline & & Mean \pm SD & Median [min-max] \\
\hline Age & & $73.6 \pm 9$ & $74[52-91]$ \\
\hline
\end{tabular}

\section{IOP Reduction, All Eyes}

Patient follow-up at 12 and 24 months postoperative across all eyes indicated a statistically significant decrease in mean IOP when compared to baseline $(n=60, P<0.001)$. The iTrack procedure resulted in a stable reduction in IOP down to $13.6 \pm 1.9 \mathrm{mmHg}$ at 12 months and $13.5 \pm 2.6$ $\mathrm{mmHg}$ at 24 months, representing a $>32 \%$ drop from baseline at all visits (Figure 1). Importantly, the procedure produced a clinically significant reduction in IOP of $\geq 25 \%$ in the large majority of eyes treated (78.4\%).

\section{Reduction in the Number of Medications, All Eyes}

Overall, there was a significant reduction in the mean number of medications at all visits across all eyes when compared to baseline (Figure 2, $P<0.001$ ). A 39\% reduction in medications was observed at 24 months $(2.8 \pm 0.9$ to $1.7 \pm 1.3$ ) compared to a $59.6 \%$ reduction observed at 12 months (2.8 \pm 0.9 to $1.1 \pm 1.1$ ). Importantly, $65 \%$ and $47 \%$ of patients were on one or fewer medications at 12 months and 24 months, respectively, compared to only $10 \%$ at

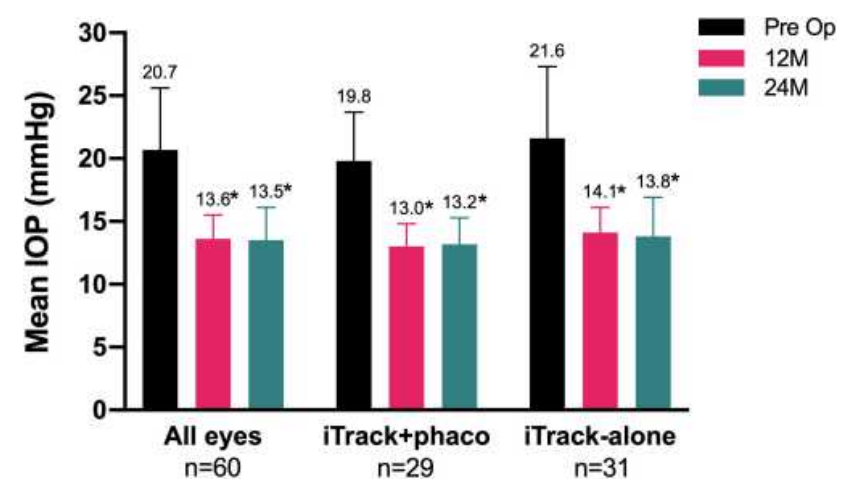

Figure I IOP reduction in iTrack+phaco and iTrack-alone groups. Mean IOP per group (and SD) at baseline and 12- and 24-month visits is represented with a histogram. The mean IOP value for each group per time-point is depicted at the top of each bar on the graph. In each group, the mean IOP at 12 and 24 months was compared to baseline and $*$ denotes statistically different values $(* P<0.001)$.

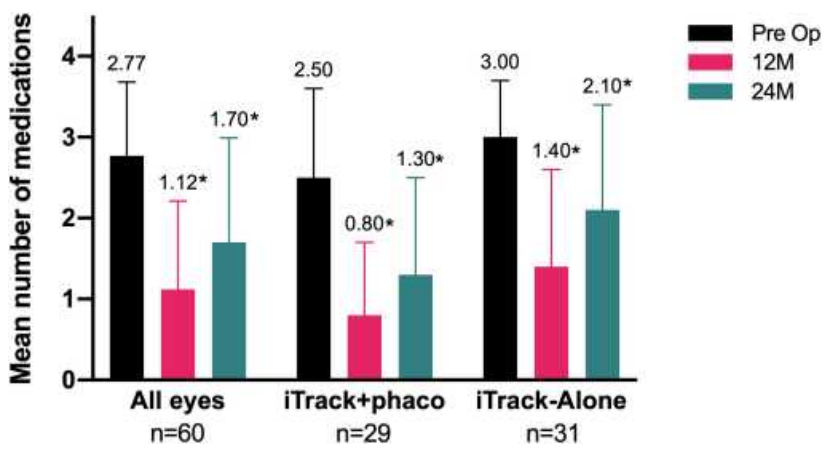

Figure 2 Mean number of medications at baseline, 12 months and 24 months, across the entire cohort, in iTrack+phaco and iTrack-alone groups. The mean number of glaucoma medications used per eye (and SD) is represented with a histogram. The mean value per group at each time point is shown at the top of each bar. In each group, there is a statistically significant decrease in number of medications used at 12 and 24 months when compared to baseline $\left({ }^{*} P<0.001\right)$.

baseline. Additionally, $25 \%$ of all eyes were free of medication at the 24-month follow-up, versus only $3.3 \%$ at baseline.

\section{IOP Reduction, iTrack-Alone Compared to iTrack+Phaco}

To evaluate the 24-month efficacy of iTrack as a standalone procedure, we compared the reduction in IOP between the iTrack-alone and the iTrack+phaco groups. Similar to the analysis in all eyes, the mean reduction in IOP was significant and stable at 12 and 24 months in both groups, compared to baseline (Figure 1, $P<0.001$ for both groups). The percentage decrease in IOP was not statistically different between the two groups at either time point, with an approximate $32 \%$ reduction in mean IOP for both groups at 12 months $(P=0.977)$ that was stable at $34 \%$ reduction for the 

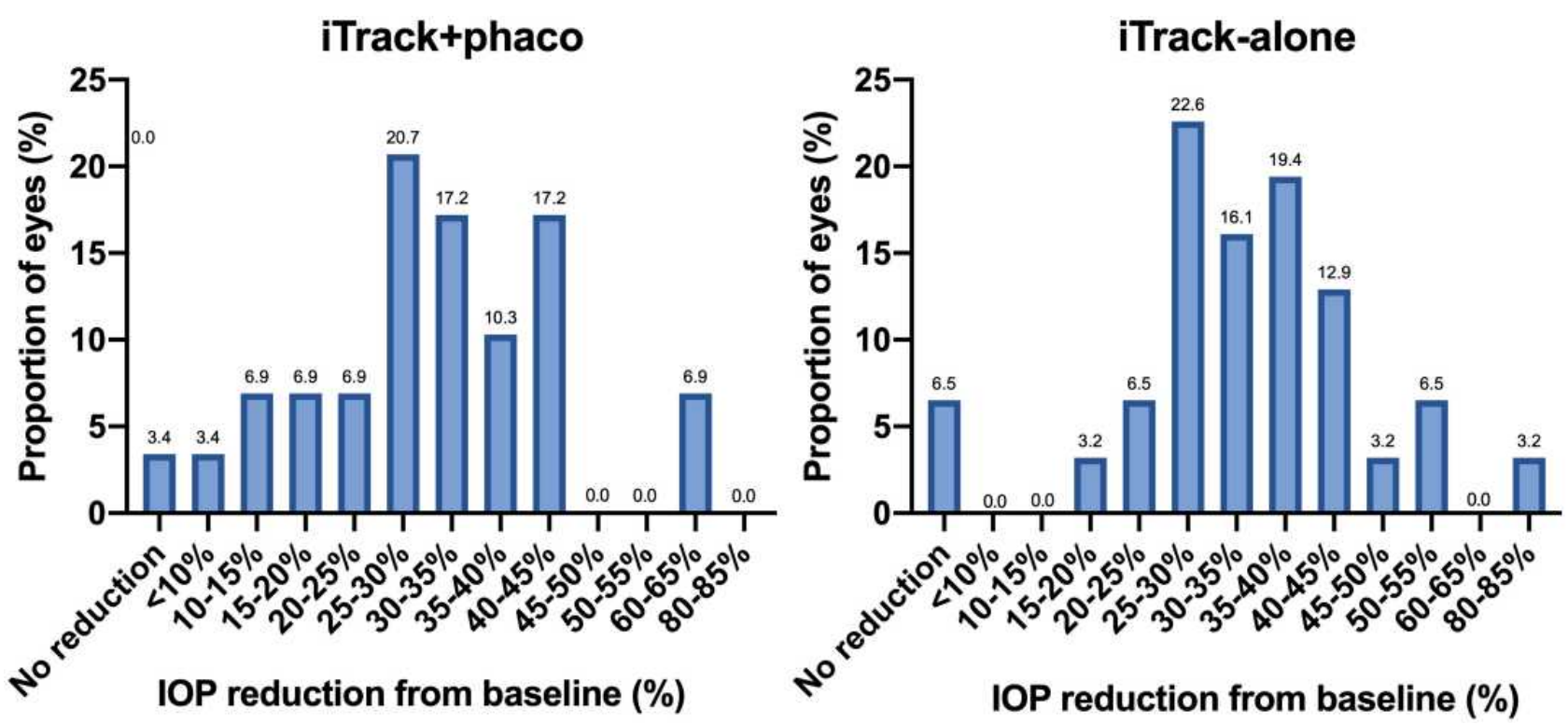

Figure 3 Stability and effectiveness of IOP reduction at 24 months postoperative in the iTrack +phaco and iTrack-alone groups. The distribution of the extent of IOP reduction from baseline $(\%)$ is represented in this figure $(n=29$ eyes an $n=31$ eyes in iTrack+phaco and iTrack-alone groups, respectively).

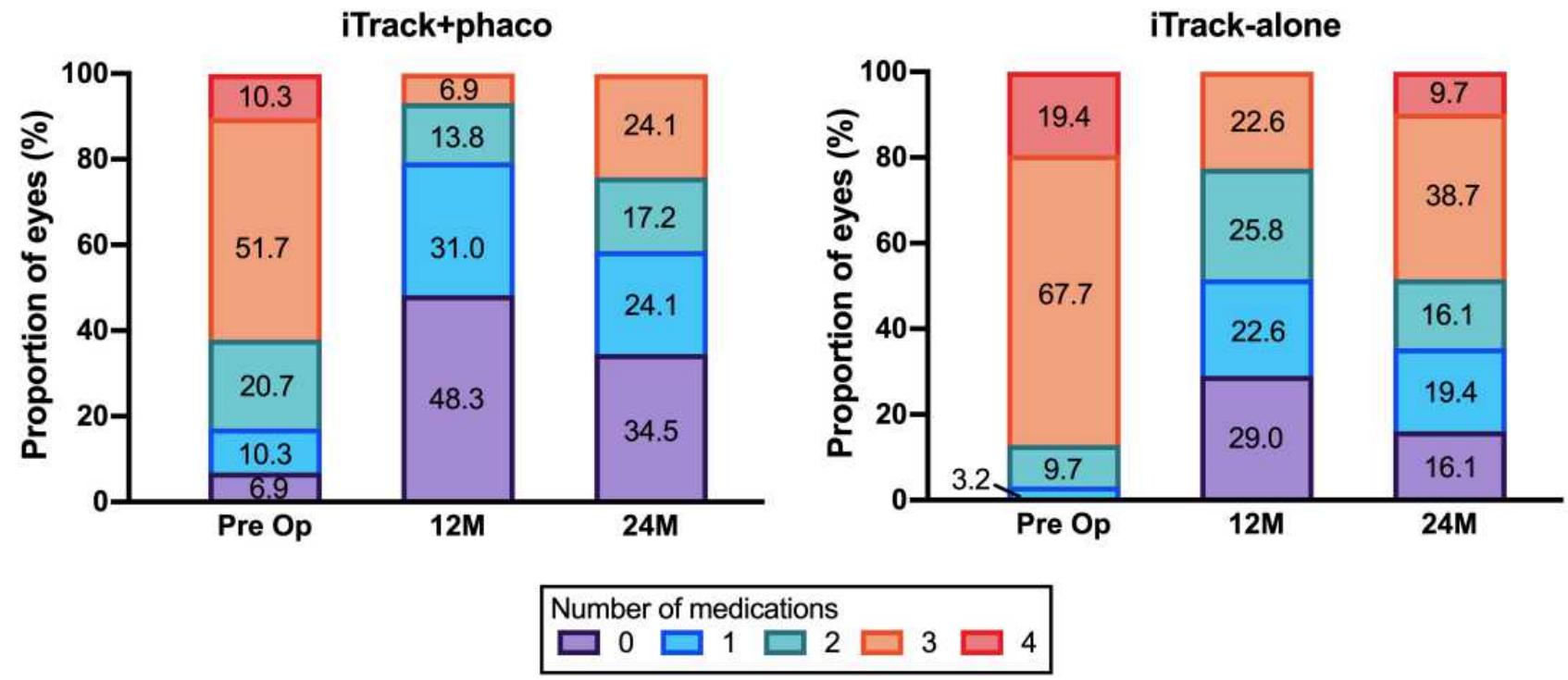

Figure 4 Proportion of eyes per number of medications. The proportion of eyes $(\%)$ per number of medications in each group $(i T r a c k+p h a c o n=29$ and $i T r a c k-a l o n e ~ n=31)$ at baseline, 12 months and 24 months is depicted.

iTrack-alone group (21.6 \pm 5.7 to $13.8 \pm 3.1)$ and $31 \%$ reduction for the iTrack+phaco group (19.8 \pm 3.9 to $13.2 \pm 2.1)$ at the 24-month visit $(P=0.512)$. Of note, the iTrack-alone group started with a higher baseline IOP than the iTrack + phaco group $(P=0.0364)(+1.20$ points $)$ and this difference was the same at each time point. Overall, a stable decrease in IOP occurred in most patients in both groups, with more than $72 \%$ of eyes in the iTrack + phaco group and more than $80 \%$ of eyes in the iTrack-alone group recording $\geq 25 \%$ reduction in IOP at 24 months (Figure 3).
Reduction in the Number of Medications, iTrack-Alone Compared to iTrack+Phaco For both the iTrack-alone and the iTrack+phaco group, there was a significant decrease in the number of medications at 12 and 24 months, compared to baseline $(P<0.001$, Figure 2). It should be noted that there was a statistically significant difference in the number of medications at baseline and at all post-op visits between groups $(P=0.004)$, with the iTrackalone group starting with a mean number of medications 0.5 
units higher than the iTrack+phaco group. The mean number of medications at baseline was $3.0 \pm 0.7$ in the iTrack-alone group and $2.5 \pm 1.1$ in the $\mathrm{i}$ Track + phaco group. At 12 months, there was a mean reduction of $51.3 \%$ in the iTrack-alone group (3.0 \pm 0.7 to $1.4 \pm 1.2$ ), compared to a mean reduction of $66.4 \%$ in the iTrack + phaco group $(2.5 \pm 1.1$ to $0.8 \pm 0.9)$. At 24 months, there was a reduction in mean medications of $31 \%$ in the iTrack-alone group $(3.0 \pm 0.7$ to $2.1 \pm 1.3)$ compared to $46.5 \%$ in the iTrack+phaco group $(2.5 \pm 1.1$ to 1.3 \pm 1.2 ), relative to baseline (Figure 2). The percentage reduction in the number of medications was not statistically different between groups at each time point $(P=0.141$ at 12 months and $P=0.182$ at 24 months). In comparing the reduction in medication across both time points, the number of medications increased by 0.7 in the iTrack-alone group and 0.5 in the iTrack+phaco group between the 12-month and 24-month visits. It is worth noting, however, that the overall reduction in medication use at 24 months, as compared to baseline, was significant in both groups $(P<0.001)$. Additionally, $16.1 \%$ of eyes in the iTrack-alone group and $34.5 \%$ in the iTrack + phaco group were free of medications at 24 months, compared to only $3.2 \%$ and $6.6 \%$ at baseline, respectively (Figure 4).

\section{Disease Severity}

In both the mild-moderate group and the severe group, there was a similar decrease in mean IOP and the mean number of medications at 12 and 24 months, compared to baseline (Table 2). In the mild-moderate group, IOP reduced from $20.8 \pm 5.3 \mathrm{mmHg}$ at baseline to $13.6 \pm 1.8$ $\mathrm{mmHg}(32.3 \%)$ and $13.5 \pm 2.5 \mathrm{mmHg}(32.7 \%)$ at 12 - and 24 months, respectively. In the severe group, IOP reduced from $20.7 \pm 4.4 \mathrm{mmHg}$ at baseline to $13.7 \pm 2.1 \mathrm{mmHg}$ $(31.8 \%)$ and $13.3 \pm 2.8 \mathrm{mmHg}(33.6 \%)$ at $12-$ and 24 months, respectively. The percentage decrease in IOP was not statistically different between the two groups ( $P=0.8962$ at 12 months and $P=0.8202$ at 24 months). At 24 months, $78.8 \%$ of eyes in the mild-moderate group and $78.3 \%$ of eyes in the severe group recorded a mean IOP of $15 \mathrm{mmHg}$ or less.

The mean reduction in medication use was statistically significant in both groups, noting that there was a greater reduction in absolute and percentage terms in the mildmoderate glaucoma group, as compared to the severe group. In the mild-moderate group, the number of medications reduced from $2.6 \pm 1.1$ at baseline to $0.9 \pm 1.1$ and $1.4 \pm 1.3$ at 12 - and 24 months, compared to a reduction from $2.9 \pm 0.7$ at baseline to $1.3 \pm 1.1$ and $2.1 \pm 1.2$ in the severe group at 12- and 24 months. At 24 months, 54.6\% of eyes in the mild-moderate group and $34.8 \%$ of patients in the severe group were on one or less medications.

\section{VA Outcomes}

Preoperative, 12-month and 24-month visual acuities are presented in Table 3 and Figure 5. Visual acuity was improved at the 24-month follow-up in all eyes, with 19 patients $(42.2 \%)$ gaining two or more lines of Snellen corrected distance visual acuity. At 24 months, $69.6 \%$ of eyes had $20 / 40$ vision or better $(P=0.0116)$, as compared to $45 \%$ of eyes at baseline. In the iTrack +Phaco group $(n=29)$ there was a significant improvement in visual acuity at the 24-month follow-up. Fourteen patients $(61.6 \%)$ gained two or more lines of Snellen CDVA and $80.8 \%$ of eyes had $20 / 40$ vision or better $(P=0.0042)$, as compared to $34.5 \%$ of eyes at baseline. In the iTrack-alone group $(n=31), 70 \%$ of eyes at the 24-month follow-up had 20/40 vision or better, compared to $64.5 \%$ at baseline $(P=0.4716)$. Three patients in the iTrack-alone group (15\%) gained two or more lines of Snellen corrected distance visual acuity at the 24-month follow-up.

Table 2 Reduction in IOP and Number of Medications According to Glaucoma Severity

\begin{tabular}{|c|c|c|c|c|c|c|}
\hline & \multicolumn{6}{|c|}{ Disease Severity } \\
\hline & \multicolumn{3}{|c|}{ Mild/Moderate } & \multicolumn{3}{|c|}{ Severe } \\
\hline & Pre Op & $12 M$ & $24 M$ & Pre Op & $12 M$ & $24 M$ \\
\hline Mean IOP in $\mathrm{mmHg} \pm \mathrm{SD}$ & $20.8 \pm 5.3$ & $13.6 \pm 1.8$ & $13.5 \pm 2.5$ & $20.7 \pm 4.4$ & $13.7 \pm 2.1$ & $13.3 \pm 2.8$ \\
\hline Number of eyes with IOP $\leq 15 \mathrm{mmHg}$ & & $29(87.9 \%)$ & $26(78.8 \%)$ & & $18(78.3 \%)$ & $18(78.3 \%)$ \\
\hline Mean number of Medications $\pm S D$ & $2.6 \pm 1.1$ & $0.9 \pm 1.1$ & $1.4 \pm 1.3$ & $2.9 \pm 0.7$ & $1.3 \pm 1.1$ & $2.1 \pm 1.2$ \\
\hline Total number of eyes & 33 & 33 & 33 & 23 & 23 & 23 \\
\hline
\end{tabular}


Table 3 Corrected Visual Acuity at Baseline and at the Follow-Up Visits

\begin{tabular}{|c|c|c|c|c|c|c|c|c|c|}
\hline & \multicolumn{9}{|c|}{ Visual Acuity: Number of Eyes per Group (\%) } \\
\hline & \multicolumn{3}{|c|}{ All eyes } & \multicolumn{3}{|c|}{ iTrack+phaco } & \multicolumn{3}{|c|}{ ¡Track-alone } \\
\hline & Pre Op & $12 M$ & 24M & Pre Op & I $2 M$ & 24M & Pre Op & I $2 M$ & 24M \\
\hline $20 / 20$ or better & $3(5.0)$ & $3(5.6)$ & $3(6.5)$ & $0(0.0)$ & $2(8.0)$ & $2(7.7)$ & $3(9.7)$ & I (3.5) & I (5.0) \\
\hline $20 / 40$ or better & $27(45.0)$ & $38(70.4)$ & $32(69.6)$ & $10(34.5)$ & $19(76.0)$ & $19(73.1)$ & $17(54.8)$ & $19(65.5)$ & $13(65.0)$ \\
\hline $20 / 60$ or better & $43(71.7)$ & $45(83.3)$ & $4 I(89.1)$ & $19(65.5)$ & $21(84.0)$ & $24(92.3)$ & $24(77.4)$ & $24(82.8)$ & $17(85.0)$ \\
\hline $20 / 100$ or better & $52(86.7)$ & 48 (88.9) & $43(93.5)$ & $24(82.8)$ & $23(92.0)$ & $25(96.2)$ & $28(90.3)$ & $25(86.2)$ & I8 (90.0) \\
\hline Tot eyes & 60 & 54 & 46 & 29 & 25 & 26 & 31 & 29 & 20 \\
\hline
\end{tabular}

\section{Surgical and Postsurgical Complications}

Overall, the frequency of surgical and postsurgical complications was low with no serious adverse events recorded. Adverse events were limited to intraoperative bleeding at the goniotomy site and postoperative microhyphema development, both of which resolved within 1 week without sequelae.

\section{Discussion}

In this retrospective and comparative case study, 360degree viscodilation of Schlemm's canal and the distal outflow system with iTrack ab-interno canaloplasty was found to be a highly effective option for sustained reduction in both IOP and glaucoma medication dependence. When performed as a standalone procedure or in combination with cataract surgery, the iTrack procedure achieved a stable $>30 \%$ IOP reduction at 24 months, with mean IOP at $13.8 \pm 3.1 \mathrm{mmHg}$ in the iTrack-alone group, compared to $13.2 \pm 2.1 \mathrm{mmHg}$ in the iTrack+phaco group.

Both iTrack-alone and iTrack+phaco groups demonstrated a significant decrease in the mean number of medications, reducing by $31.4 \%$ in the iTrack-alone group and $46.5 \%$ in the iTrack+phaco group, as compared to baseline. The ability to deploy iTrack as a standalone procedure, and in combination with cataract surgery, supports its versatility in the glaucoma treatment algorithm.

It is worth noting that the iTrack+phaco results may be confounded by the IOP-lowering effect of cataract surgery. Cataract surgery is known to lower IOP in some glaucoma patients, particularly those with a higher preoperative IOP. ${ }^{17}$ The physiological reasons behind this phenomenon

\section{iTrack+phaco}

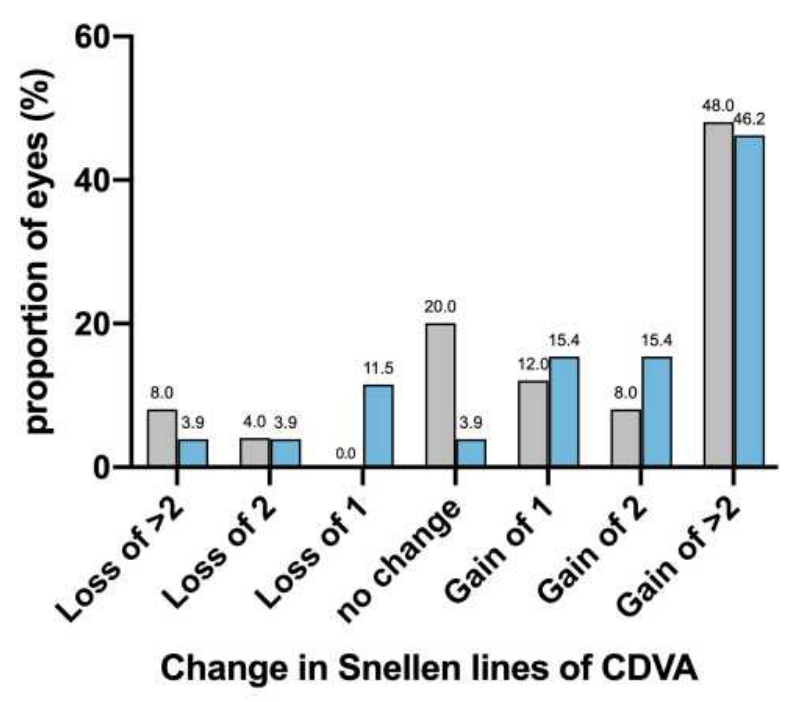

Figure 5 Visual acuity. For each group, corrected distance visual acuity is represented as loss or gain of lines of Snellen at the 12- and 24-month follow-up visits, compared to baseline. Visual acuity data at 12 months were available for $n=25$ eyes in the iTrack+phaco group and $n=29$ in the iTrack-alone group. At 24 months, values were available for $n=26$ eyes in the iTrack+phaco group and $n=20$ in the iTrack-alone group. 
are not entirely clear, although it has been suggested that cataract surgery may impact the anterior chamber depth and the trabecular-iris angle, thus contributing to reduced outflow resistance. ${ }^{18}$ Regardless, the efficacy and safety of iTrack in the present study were the same when performed combined with cataract surgery or as a standalone procedure, lending evidence to its utility.

The 24-month results of the present study are in line with previously reported 12-month results. ${ }^{10,19,20}$ A 2018 study by Gallardo et al demonstrated a mean IOP reduction of $32.8 \%$ $(P<0.001)$ in standalone cases $(\mathrm{n}=41)$ and $31.7 \%(P<$ $0.001)$ in combined cataract surgery cases $(\mathrm{n}=34)$ respectively, at 12 months. In a 2018 study by Koerber et al, ${ }^{19}$ mean IOP reduced from $18.8 \pm 5.63$ at baseline $(n=23)$ to $14.73 \pm$ $2.97 \mathrm{mmHg}$ at 12 months $(\mathrm{n}=11)$. Patient reliance on medications was also reduced, with the mean number of medications falling from 1.69 at baseline $(n=23)$ to 0.21 at the last followup visit $(n=11)$. A 2020 study by Kazerounian et al ${ }^{20}$ demonstrated a reduction in mean IOP of $32.5 \%$ from $20.24 \mathrm{mmHg}$ $\pm 5.92(\mathrm{n}=25)$ at baseline to $13.67 \mathrm{mmHg} \pm 2.15(\mathrm{n}=21$, $P<0.001)$ at 24 months following iTrack ab-interno canaloplasty. Medication reduction was also significant, falling from $1.92 \pm 1.04$ at baseline to $0.05 \pm 0.23$ at 24 months. At 24 months, $80 \%$ of the patients were on zero medications. The ability of iTrack ab-interno canaloplasty to maintain the reduction in IOP and medications out to 24 months is an important finding, as it has been previously suggested that the clinical effect of the procedure may diminish over time. In contrast, the results of the present study recommend that the combined process of 360-degree catheterization and viscodilation can effectively reduce IOP and medications over the long term, without the need for a permanent implant. Further investigations should be undertaken to elucidate the mechanisms of action by which ab-interno canaloplasty can achieve a long-term reduction in IOP and medication use.

The role of viscodilation during ab-interno canaloplasty also warrants further investigation. In the present study, approximately 36 microboluses of OVD were delivered over 360 degrees of the canal. This is in contrast to the traditional ab-externo canaloplasty procedure, in which viscodilation comprises a maximum of 12-15 microboluses. With ab-interno canaloplasty the anterior chamber is pressurized with OVD and thereby creates a tamponade effect on the cornea, making it feasible to viscodilate quite aggressively with minimal if any risk of Descemet's detachment. During the ab-interno procedure, the process of viscodilation is postulated to improve the outflow facility via dilatation of the canal, and via the removal of herniations of the trabecular meshwork into the collector channel ostia.

It is also worth addressing the efficacy of iTrack abinterno canaloplasty based on the grade of disease severity. In the present study, iTrack was shown to be effective in cases of mild-moderate and severe glaucoma, achieving similar reductions in mean IOP at the 24-month mark at $32.7 \%$ and $33.6 \%$, respectively. Noting that MIGS procedures were first conceived for use in the treatment of mildmoderate glaucoma, the ability to also deploy iTrack abinterno canaloplasty in cases of severe glaucoma offers significant utility in potentially deferring or eliminating the need for invasive surgical options, such as trabeculectomy and tube shunt surgery. Moreover, the fact that iTrack addresses multiple sites of outflow resistance ${ }^{12-15}$ may also support its deployment across a broad spectrum of POAG patients. The majority of MIGS are focal in their approach and target the proximal portion of the outflow system only. If the primary site of resistance to aqueous humor outflow is the trabecular meshwork, these procedures would bypass the site of obstruction and facilitate access to Schlemm's canal and the distal outflow system. However, if obstruction is located distally, then the efficacy of these procedures will likely be limited. iTrack, on the other hand, has the advantage of addressing all points of resistance. ${ }^{11-14}$

Limitations of the present study include that it was a single-center, retrospective case series with a relatively small population cohort. The study is limited by the characteristic confines of retrospective research, including selection bias. It should be noted that the data presented include a consistent cohort of patients that had available IOP at 24 months. Patients who were lost to follow-up or failed prior were excluded from this reported population.

\section{Conclusion}

A comprehensive MIGS procedure that can be performed as a standalone procedure or combined with cataract surgery, and in cases of mild, moderate and severe glaucoma, iTrack ab-interno canaloplasty has a highly versatile potential. The 24-month follow-up data presented here show that it can be used effectively and safely to lower both IOP and the number of antiglaucoma medications. Thus, inclusion of iTrack ab-interno canaloplasty in the glaucoma treatment algorithm can help reduce or eliminate the need for antiglaucoma medications and delay more aggressive surgeries without limiting the use of future interventions. The promising results seen with this 
relatively small group of patients confirm the need for prospective, multicenter trials with a larger patient cohort.

\section{Abbreviations}

POAG, primary open-angle glaucoma; TM, trabecular meshwork; CDVA, corrected distance visual acuity.

\section{Acknowledgments}

The author thanks Nicole Sanchez for data collection. Nova Eye Medical has provided statistical analysis and manuscript preparation support.

\section{Disclosure}

Dr Mark J Gallardo is clinical investigator, consultant, speaker for Nova Eye Medical; clinical investigator, consultant, speaker for Sight Sciences; clinical investigator, consultant, speaker for Ivantis; clinical investigator, consultant, speaker for Glaukos; clinical investigator, consultant, speaker for Allergan. The author reports no other conflicts of interest in this work.

\section{References}

1. Quigley HA, Broman AT. The number of people with glaucoma worldwide in 2010 and 2020. Br J Ophthalmol. 2006;90(3):262-267. doi:10.1136/bjo.2005.081224

2. American Academy of Ophthalmology. Preferred Practice Pattern ${ }^{\circledR}$ Guidelines. Primary Open-Angle Glaucoma. San Francisco, CA: American Academy of Ophthalmology; 2015.

3. Kaur D, Gupta A, Singh G. Perspectives on quality of life in glaucoma. J Curr Glaucoma Pract. 2012;6(1):9-12. doi:10.5005/jpjournals-10008-1101

4. Zhang X, Vadoothker S, Munir WM, Saeedi O. Ocular surface disease and glaucoma medications: a clinical approach. Eye Contact Lens. 2019;45(1):11-18. doi:10.1097/ICL.0000000000000544

5. Swaminathan SS, Dong-Jin Oh DJ, Hyung M, et al. Aqueous outflow: segmental and distal flow. $J$ Cataract Refract Surg. 2014;40 (8):1263-1272. doi:10.1016/j.jcrs.2014.06.020

6. Hong J, Xu J, Wei A, et al. Spectral-domain optical coherence tomographic assessment of Schlemm's canal in Chinese subjects with primary open-angle glaucoma. Ophthalmology. 2013;120(4):709-715. doi:10.1016/j.ophtha.2012.10.008
7. Allingham RR, de Kater AW, Ethier CR. Schlemm's canal and primary open angle glaucoma: correlation between Schlemm's canal dimensions and outflow facility. Exp Eye Res. 1996;62(1):101-109. doi:10.1006/exer.1996.0012

8. Hann CR, Vercnocke AJ, Bentley MD, Jorgensen SM, Fautsch MP. Anatomic changes in Schlemm's canal and collector channels in normal and primary open-angle glaucoma eyes using low and high perfusion pressures. Invest Ophthalmol Vis Sci. 2014;55(9):58 34-5841. doi:10.1167/iovs.14-14128

9. Cha ED, Xu J, Gong H. Variations in active areas of aqueous humor outflow through the trabecular outflow pathway. Presented at ARVO 2015. Invest Ophthalmol Vis Sci. 2015;56:4850.

10. Gallardo MJ, Supnet RA, Ahmed IIK. Viscodilation of Schlemm's canal for the reduction of IOP via an ab-interno approach. Clin Ophthalmol. 2018;12:2149-2155. doi:10.2147/OPTH.S177597

11. Körber N. Canaloplasty ab interno - a minimally invasive alternative. Klin Monbl Augenheilkd. 2017;234(8):991-995. doi:10.1055/s-0042123829

12. Stegmann R, Pienaar A, Grieshaber MC. Schlemm's canal surgery: restoring physiological aqueous outflow. In: Grieshaber MC, Orgul S, Flammer J, editors. Glaucoma Therapy-State of the Art. Basel. Switzerland: Association for Continuing Education in Ophthalmology; 2009:113-120.

13. Stegmann R, Pienaar A, Miller D. Viscocanalostomy for open-angle glaucoma in black African patients. J Cataract Refract Surg. 1999;25 (3):316-322. doi:10.1016/S0886-3350(99)80078-9

14. Grieshaber MC, Pienaar A, Olivier J, Stegmann R. Clinical evaluation of the aqueous outflow system in primary open-angle glaucoma for canaloplasty. Invest Ophthalmol Vis Sci. 2010;51(3):1498-1504. doi:10.1167/iovs.09-4327

15. Smit BA, Johnstone MA. Effects of viscoelastic injection into Schlemm's canal in primate and human eyes: potential relevance to viscocanalostomy. Ophthalmology. 2002;109(4):786-792. doi:10.10 16/S0161-6420(01)01006-5

16. Hodapp E, Parrish RK II, Anderson DR. Clinical Decisions in Glaucoma. St Louis: The CV Mosby Co; 1993:5.

17. Berdahl JP. Cataract surgery to lower intraocular pressure. Middle East Afr J Ophthalmol. 2009;16(3):119-122. doi:10.4103/0974-9233. 56222

18. Rekas M, Barchan-Kucia K, Konopin'ska J, Mariak Z, Zarnowski T. Analysis and modeling of anatomical changes of the anterior segment of the eye after cataract surgery with consideration of different phenotypes of eye structure. Curr Eye Res. 2014;1-10.

19. Körber N. Ab interno canaloplasty for the treatment of glaucoma: a case series study. Spektrum Augenheilkd. 2018;32:223227. doi:10. 1007/s00717-018-0416-7

20. Kazerounian S, Zimbelmann M, Lörtscher M, Hommayda S, Tsirkinidou I, Müller M. Canaloplasty ab interno (AbiC) - 2-yearresults of a novel Minimally Invasive Glaucoma Surgery (MIGS) Technique. Klin Monbl Augenheilkd. 2020. doi:10.1055/a-1250-8431
Clinical Ophthalmology

\section{Publish your work in this journal}

Clinical Ophthalmology is an international, peer-reviewed journal covering all subspecialties within ophthalmology. Key topics include: Optometry; Visual science; Pharmacology and drug therapy in eye diseases; Basic Sciences; Primary and Secondary eye care; Patient Safety and Quality of Care Improvements. This journal is indexed on PubMed
Central and CAS, and is the official journal of The Society of Clinical Ophthalmology (SCO). The manuscript management system is completely online and includes a very quick and fair peer-review system, which is all easy to use. Visit http://www.dovepress.com/ testimonials.php to read real quotes from published authors. 